\title{
A Nonignorable Attrition in Pairwise Randomized Experiments
}

\author{
Kentaro Fukumoto
}

Department of Political Science, Gakushuin University, 1-5-1 Mejiro, Toshima, Tokyo 171-8588, Japan. Email:Kentaro.Fukumoto@gakushuin.ac.jp

\begin{abstract}
In pairwise randomized experiments, what if the outcomes of some units are missing? One solution is to delete missing units (the unitwise deletion estimator, UDE). If attrition is nonignorable, however, the UDE is biased. Instead, scholars might employ the pairwise deletion estimator (PDE), which deletes the pairmates of missing units as well. This study proves that the PDE can be biased but more efficient than the UDE and, surprisingly, the conventional variance estimator of the PDE is unbiased in a super-population. I also propose a new variance estimator for the UDE and argue that it is easier to interpret the PDE as a causal effect than the UDE. To conclude, I recommend the PDE rather than the UDE.
\end{abstract}

Keywords: local average treatment effect, matched-pair design, not missing at random, principal effect, potential outcome

\section{Introduction}

In pairwise randomized experiments, researchers collect pairs of units, where the two units in each pair share the exact or similar values of matched-on variables, and randomly assign treatment to one unit in each pair (Imbens and Rubin 2015, ch. 10). The difference-in-means of outcomes between the treated and control groups estimates the average treatment effect (hereafter, ATE) on the outcome without bias. This design is an efficient tool to enable pretreatment balance, in particular when the number of units is small (Donner and Klar 2000, 32).

Nonetheless, a major problem of pairwise randomized experiments is attrition; the outcomes

Political Analysis (2022) vol. 30: 132-141

DOI: $10.1017 /$ pan.2020.51

Published

9 March 2021

Corresponding author

Kentaro Fukumoto

Edited by

Jeff Gill

(c) The Author(s) 2021. Published by Cambridge University Press on behalf of the Society for

Political Methodology. This is an Open Access article, distributed under the terms of the Creative Commons AttributionNonCommercial-NoDerivatives licence

(https://creativecommons.org/ licenses/by-nc-nd/4.0/), which permits non-commercial re-use, distribution, and reproduction in any medium, provided the original work is unaltered and is properly cited. The written permission of Cambridge University Press must be obtained for commercial reuse or in order to create a derivative work. of some units are sometimes missing (Donner and Klar 2000, 40; Glennerster and Takavarasha 2013, 159; Hayes and Moulton 2009, 72-74). Moreover, attrition might be nonignorable, that is, it may be related to the value of an outcome (Allison 2002, 4-5). The typical solutions to attrition, inverse probability weighting and (multiple) imputation techniques, do not work in this situation (Allison 2002; Little and Rubin 2002). Accordingly, if analysts are not satisfied with bounds (Imai and Jiang 2018), they will usually employ one of the following two methods.

The first, but naive, approach to attrition is the unitwise deletion estimator (UDE). That is, analysts delete only missing observations and apply the difference-in-means estimator to all the remaining units to estimate the ATE (Glennerster and Takavarasha 2013, 159). It is, however, well known that the UDE can be biased if attrition is nonignorable (e.g., Little and Rubin 2002, 41-44).

The second textbook tool to address attrition is the pairwise deletion estimator (PDE), which deletes missing units as well as the other units in the same pairs and calculates the difference-in-means by using units in the remaining pairs only (Donner and Klar 2000, 40; Imai, King, and Nall 2009, 44). The PDE protects the balance of matched-on variables, but it is not sufficient to retain the distribution of treatment effects across units and thus guarantee unbiased estimation of the ATE. Rather, as Dunning (2011) and Gerber and Green (2012) warn, the PDE can be biased. Scholars may also suspect that since the PDE discards more units, it will be less efficient than the UDE (Hayes and Moulton 2009, 73-74). 
Even though both the UDE and the PDE can be biased, we should use one of them given no other option. Previous studies do not examine the bias and variance of these estimators in this situation. This paper takes the design of pairwise randomization seriously, derives the properties of the two estimators and their variance estimators, and recommends the PDE rather than the UDE. Proof of the propositions, detailed comments on them, and an application example are available in the Supplementary Material.

\section{Finite Sample}

\subsection{Setting}

Suppose that there are $N(\geq 2)$ pairs and each pair is composed of two units. Let $Y_{i j}, R_{i j}$, and $X_{i j}$ denote the realized outcome, realized response, and treatment status, respectively, for unit $i$ of pair $j$ where $i=1,2$ and $j=1,2, \ldots, N . R_{i j}$ is equal to one (zero) when $Y_{i j}$ is observed (missing). $X_{i j}$ is equal to one (zero) when unit $i$ of pair $j$ is assigned to the treated (control) group. Since this is a pairwise randomized experiment, for every pair $j$, either $X_{1 j}=1, X_{2 j}=0$ or $X_{1 j}=0, X_{2 j}=1$ holds.

I make the stable unit treatment value assumption (Imbens and Rubin 2015). $Y_{i j}(1)$ and $R_{i j}(1)$ denote the potential outcome and response for unit $i$ of pair $j$ if treatment is assigned to the unit $\left(X_{i j}=1\right)$, respectively. $R_{i j}(1)$ is equal to one (zero) when $Y_{i j}=Y_{i j}(1)$ is observed (missing) in the case of treatment. $Y_{i j}(0)$ and $R_{i j}(0)$ are defined similarly for the case of control.

The main estimand in this section, the finite sample ATE, is denoted by $\tau \equiv E\left\{Y_{i j}(1)-Y_{i j}(0)\right\} \equiv$ $\frac{1}{2 N} \sum_{j=1}^{N} \sum_{i=1}^{2}\left\{Y_{i j}(1)-Y_{i j}(0)\right\}$. The full sample estimator is denoted by $\hat{\tau}_{F} \equiv E\left(Y_{i j} \mid X_{i j}=1\right)-E\left(Y_{i j} \mid\right.$ $\left.X_{i j}=0\right)$. This estimator is not available in the case of attrition. Rather, analysis of $\hat{\tau}_{F}$ provides reference benchmarks against which this study compares the properties of the UDE and PDE.' The UDE is denoted by $\hat{\tau}_{U} \equiv E\left(Y_{i j} \mid X_{i j}=1, R_{i j}=1\right)-E\left(Y_{i j} \mid X_{i j}=0, R_{i j}=1\right)$. Obviously, it can be defined only when $N_{t} \equiv \sum_{j=1}^{N} \sum_{i=1}^{2} X_{i j} R_{i j} \geq 1$ and $N_{c} \equiv \sum_{j=1}^{N} \sum_{i=1}^{2}\left(1-X_{i j}\right) R_{i j} \geq 1$. The PDE is denoted by $\hat{\tau}_{P} \equiv E\left(Y_{i j} \mid X_{i j}=1, R_{1 j}=R_{2 j}=1\right)-E\left(Y_{i j} \mid X_{i j}=0, R_{1 j}=R_{2 j}=1\right)$. It can be defined only when $N_{t c} \equiv \sum_{j=1}^{N} R_{1 j} R_{2 j} \geq 1$.

In order to clarify the properties of estimators in the propositions below, for $x=0,1$, this study denotes the between-pair and within-pair deviations of potential outcome by $\beta_{i j}(x) \equiv\left\{Y_{1 j}(x)+\right.$ $\left.Y_{2 j}(x)\right\} / 2-E\left\{Y_{i^{\prime} j^{\prime}}(x)\right\}$ and $\omega_{i j}(x) \equiv Y_{i j}(x)-\left\{Y_{1 j}(x)+Y_{2 j}(x)\right\} / 2$

This study assumes pairwise randomization of treatment assignment. That is, each $X_{1 j}$ is ignorable and independent, and $\operatorname{Pr}\left(X_{1 j}=1\right)=1 / 2$. In addition, the following three assumptions of potential responses are optional; I will invoke one of them in each of the propositions below. "FS" stands for the "finite sample." First, I specify the assumption under which no outcomes are missing and thus $\hat{\tau}_{F}$ is available.

$$
\text { AsSUmPtION } 1 \text { (No Attrition: FS) } \forall i, j, R_{i j}(1)=R_{i j}(0)=1 \text {. }
$$

Second, we assume a perfect match in the sense that the potential responses are the same between the units in every pair. For instance, in the cases of (monozygotic) twins and littermates of the same sex, matched-on variables (e.g., [part of] DNA) may completely explain the missingness pattern. Under this assumption, $N_{t}$ and $N_{c}$ are constant regardless of treatment assignment, and the properties of $\hat{\tau}_{U}$ can be expressed simply enough to understand its essence.

$$
\text { ASSUMPTION } 2 \text { (Unitwise Matched Attrition: FS) } \forall j, R_{1 j}(1)=R_{2 j}(1), R_{1 j}(0)=R_{2 j}(0) \text {. }
$$

Finally, we consider the assumption under which $N_{t c}$ is constant regardless of treatment assignment, when the properties of $\hat{\tau}_{P}$ can be presented concisely. This assumption holds if (but not

1 Imai (2008) and Imbens and Rubin (2015, ch. 10) have already established the properties of $\hat{\tau}_{F}$ except for Propositions 3 (1) and $3^{*}(1)$. 
only if) either (or both) of the following two scenarios is true in every pair $j$. The first scenario is when attrition is unitwise matched $\left(R_{1 j}(1)=R_{2 j}(1), R_{1 j}(0)=R_{2 j}(0)\right)$. (Therefore, Assumption 2 always leads to Assumption 3 but not vice versa.) The second scenario is when each unit is either an "always-reporter" $\left(R_{i j}(1)=R_{i j}(0)=1\right)$ or a "never-reporter" $\left(R_{i j}(1)=R_{i j}(0)=0\right)$ (Gerber and Green 2012, 225). For instance, in the cases of blind tests, subliminal stimuli, and administrative records, it is likely that $R_{i j}(1)=R_{i j}(0)$.

AsSUMPTION 3 (Pairwise Matched Attrition: FS) $\forall j, R_{1 j}(1) R_{2 j}(0)=R_{1 j}(0) R_{2 j}(1)$.

\subsection{Bias}

In this section, the operator $\mathbb{E}(\cdot)$ takes expectation over the random assignment of the treatment. Now, I present the bias of each ATE estimator.

\section{PROPOSITION 1 (Bias of ATE Estimators: FS)}

(1) Under Assumption 1, $\mathbb{E}\left(\hat{\tau}_{F}\right)-\tau=0$.

(2) Under Assumption 3 and $N_{t c} \geq 1, \mathbb{E}\left(\hat{\tau}_{P}\right)-\tau=E\left\{\beta_{i j}(1)-\beta_{i j}(0) \mid R_{1 j}(1)=R_{2 j}(1)=R_{1 j}(0)=\right.$ $\left.R_{2 j}(0)=1\right\}$.

(3) UnderAssumption 2 and $N_{t}, N_{c} \geq 1, \mathbb{E}\left(\hat{\tau}_{U}\right)-\tau=E\left\{\beta_{i j}(1) \mid R_{i j}(1)=1\right\}-E\left\{\beta_{i j}(0) \mid R_{i j}(0)=1\right\}$.

A few remarks are in order. First, unless we assume ignorable attrition, not only $\hat{\tau}_{U}$ but also $\hat{\tau}_{P}$ are biased for $\tau$. Second, $\hat{\tau}_{P}$ has a causal interpretation under a weaker assumption than $\hat{\tau}_{U}$. Under Assumption 3 and $N_{t c} \geq 1$, I define a kind of local average treatment effect (LATE) of "alwaysreporting pairs" by $\tau_{P} \equiv E\left\{Y_{i j}(1)-Y_{i j}(0) \mid R_{1 j}(1)=R_{2 j}(1)=R_{1 j}(0)=R_{2 j}(0)=1\right\} .{ }^{2}$ This is a causal effect because it is a principal effect (Frangakis and Rubin 2002) where the corresponding principal stratum is a set of such pairs that $R_{1 j}(1)=R_{2 j}(1)=R_{1 j}(0)=R_{2 j}(0)=1$. It follows that $\hat{\tau}_{P}$ is unbiased for $\tau_{P}: \mathbb{E}\left(\hat{\tau}_{P}\right)-\tau_{P}=0$. This argument may correctly remind readers of instrumental variable estimation for noncompliance cases. By contrast, even under Assumption 2 and $N_{t}, N_{c} \geq 1$, it is difficult to interpret $\hat{\tau}_{U}$ as a causal effect unless $R_{i j}(1)=R_{i j}(0)$ for all $i$ and $j$, in which case $\hat{\tau}_{U}$ is reduced to $\hat{\tau}_{P}$.

\subsection{Variance}

In this section, $\operatorname{Var}(\cdot)$ and $\operatorname{Cov}(\cdot, \cdot)$ denote the finite sample variance and covariance, respectively, and the operator $\mathbb{V}^{2}(\cdot)$ takes variance over the random assignment of the treatment. Here are the variances of the three ATE estimators.

PROPOSITION 2 (Variance of ATE Estimators: FS)

(1) Under Assumption 1,

$$
\mathbb{V}^{2}\left(\hat{\tau}_{F}\right)=\frac{1}{N}\left[\operatorname{Var}\left\{\omega_{i j}(1)\right\}+\operatorname{Var}\left\{\omega_{i j}(0)\right\}+2 \operatorname{Cov}\left\{\omega_{i j}(1), \omega_{i j}(0)\right\}\right] .
$$

(2) Under Assumption 3 and $N_{t c} \geq 1$,

$$
\begin{aligned}
\mathbb{V}^{2}\left(\hat{\tau}_{P}\right)= & \frac{1}{N_{t c}}\left[\operatorname{Var}\left\{\omega_{i j}(1) \mid R_{1 j}(1)=R_{2 j}(1)=R_{1 j}(0)=R_{2 j}(0)=1\right\}\right. \\
& +\operatorname{Var}\left\{\omega_{i j}(0) \mid R_{1 j}(1)=R_{2 j}(1)=R_{1 j}(0)=R_{2 j}(0)=1\right\} \\
& \left.+2 \operatorname{Cov}\left\{\omega_{i j}(1), \omega_{i j}(0) \mid R_{1 j}(1)=R_{2 j}(1)=R_{1 j}(0)=R_{2 j}(0)=1\right\}\right] .
\end{aligned}
$$

2 Imai and Jiang (2018) calls it "the average treatment effect for always-observed pairs." They derive bounds of $\hat{\tau}_{P}$ without making Assumption 3 and propose a sensitivity analysis. Their study complements the present paper. 
(3) Under Assumption 2 and $N_{t}, N_{c} \geq 1$,

$$
\begin{aligned}
\mathbb{V}^{2}\left(\hat{\tau}_{U}\right)= & \frac{1}{N_{t}} \operatorname{Var}\left\{\omega_{i j}(1) \mid R_{i j}(1)=1\right\}+\frac{1}{N_{c}} \operatorname{Var}\left\{\omega_{i j}(0) \mid R_{i j}(0)=1\right\} \\
& +\frac{2 N_{t c}}{N_{t} N_{c}} \operatorname{Cov}\left\{\omega_{i j}(1), \omega_{i j}(0) \mid R_{1 j}(1)=R_{2 j}(1)=R_{1 j}(0)=R_{2 j}(0)=1\right\} .
\end{aligned}
$$

It is worth while mentioning that even though the number of units used for estimation is not smaller for $\hat{\tau}_{U}$ than for $\hat{\tau}_{P}$ (i.e., $N_{t}, N_{c} \geq N_{t c}$ ), $\hat{\tau}_{U}$ can be less efficient than $\hat{\tau}_{P}$ (i.e., $\mathbb{V}^{2}\left(\hat{\tau}_{U}\right)>\mathbb{V}^{2}\left(\hat{\tau}_{P}\right)$ ) if $\operatorname{Var}\left\{\omega_{i j}(x) \mid R_{1 j}(x)=R_{2 j}(x)=1, R_{1 j}(1-x)=R_{2 j}(1-x)=0\right\}$ is sufficiently larger than $\operatorname{Var}\left\{\omega_{i j}(x) \mid\right.$ $\left.R_{1 j}(1)=R_{2 j}(1)=R_{1 j}(0)=R_{2 j}(0)=1\right\}$ for $x=0,1$.

\subsection{Variance Estimator}

How should we estimate the variances of these three ATE estimators? For certain situations, some scholars advocate "breaking the matches" (Lynn and McCulloch 1992), namely, analyzing data from pairwise randomized experiments as if they are completely randomized experiments. Thus, I begin with the Neyman variance estimator. Suppose that Assumption 1 holds. For $x=0,1$, if $X_{i j}=x$, a natural estimator of $\omega_{i j}(x)$ is $\hat{\omega}_{i j}(x) \equiv Y_{i j}-E\left(Y_{i j} \mid X_{i j}=x\right)$. Accordingly, we may estimate $\operatorname{Var}\left\{\omega_{i j}(x)\right\}$ in the first and second terms of the equation in Proposition 2 (1) by

$$
\frac{N}{N-1} \operatorname{Var}\left\{\hat{\omega}_{i j}(x) \mid X_{i j}=x\right\}=\frac{N}{N-1} \operatorname{Var}\left(Y_{i j} \mid X_{i j}=x\right)
$$

The third term of the equation in Proposition 2 (1) "is generally impossible to estimate empirically because we never observe both $Y_{i j}(1)$ and $Y_{i j}(0)$ for the same unit" (Imbens and Rubin 2015, 92). Thus, if we dismiss the third term, we derive the Neyman variance estimator of $\hat{\tau}_{F}$ as

$$
\widehat{\mathbb{V}}^{\text {Neyman }}\left(\hat{\tau}_{F}\right) \equiv \frac{1}{N-1}\left\{\operatorname{Var}\left(Y_{i j} \mid X_{i j}=1\right)+\operatorname{Var}\left(Y_{i j} \mid X_{i j}=0\right)\right\}
$$

Similarly, without Assumption 1, if $N_{t c} \geq 2$ or $N_{t}, N_{c} \geq 2$, we can derive the Neyman variance estimators of $\hat{\tau}_{P}$ or $\hat{\tau}_{U}$ as

$$
\begin{aligned}
& \widehat{\mathbb{V}}^{\text {Neyman }}\left(\hat{\tau}_{P}\right) \equiv \frac{1}{N_{t c}-1}\left\{\operatorname{Var}\left(Y_{i j} \mid X_{i j}=1, R_{1 j}=R_{2 j}=1\right)+\operatorname{Var}\left(Y_{i j} \mid X_{i j}=0, R_{1 j}=R_{2 j}=1\right)\right\} \\
& \widehat{\mathbb{V}}^{\text {Neyman }}\left(\hat{\tau}_{U}\right) \equiv \frac{1}{N_{t}-1} \operatorname{Var}\left(Y_{i j} \mid X_{i j}=1, R_{i j}=1\right)+\frac{1}{N_{c}-1} \operatorname{Var}\left(Y_{i j} \mid X_{i j}=0, R_{i j}=1\right),
\end{aligned}
$$

respectively.

Nonetheless, researchers do not have to give up estimating the third terms in the equations in Proposition 2. A merit of pairwise randomized experiments is that even if $X_{1 j}=1, R_{2 j}=1$, analysts may estimate $\omega_{1 j}(0)$ by $\hat{\omega}_{1 j}(0) \equiv-\hat{\omega}_{2 j}(0)$ because $\omega_{1 j}(0)=-\omega_{2 j}(0)$. This finding is the most important contribution of this study. Therefore, when $N_{t c} \geq 2$, we may estimate $\operatorname{Cov}\left\{\omega_{i j}(1), \omega_{i j}(0) \mid\right.$ $\left.R_{1 j}(1)=R_{2 j}(1)=R_{1 j}(0)=R_{2 j}(0)=1\right\}$ in the third terms by

$$
\frac{N_{t c}}{N_{t c}-1} \operatorname{Cov}\left\{\hat{\omega}_{i j}(1), \hat{\omega}_{i j}(0) \mid R_{1 j}=R_{2 j}=1\right\}=-\frac{N_{t c}}{N_{t c}-1} \operatorname{Cov}\left(Y_{t j}, Y_{c j} \mid R_{1 j}=R_{2 j}=1\right)
$$


where $Y_{t j}=\sum_{i=1}^{2} X_{i j} Y_{i j}$ and $Y_{c j}=\sum_{i=1}^{2}\left(1-X_{i j}\right) Y_{i j}$. Accordingly, when $N_{t c} \geq 2$, I propose the adjusted Neyman variance estimators as

$$
\begin{aligned}
\widehat{\mathbb{V}}^{\text {Adj-Neyman }}\left(\hat{\tau}_{F}\right) \equiv & \frac{1}{N-1}\left\{\operatorname{Var}\left(Y_{i j} \mid X_{i j}=1\right)+\operatorname{Var}\left(Y_{i j} \mid X_{i j}=0\right)-2 \operatorname{Cov}\left(Y_{t j}, Y_{c j}\right)\right\} \\
= & \frac{1}{N-1} \operatorname{Var}\left(Y_{t j}-Y_{c j}\right) \\
\widehat{\mathbb{V}}^{\text {Adj-Neyman }}\left(\hat{\tau}_{P}\right) \equiv & \frac{1}{N_{t c}-1}\left\{\operatorname{Var}\left(Y_{i j} \mid X_{i j}=1, R_{1 j}=R_{2 j}=1\right)+\operatorname{Var}\left(Y_{i j} \mid X_{i j}=0, R_{1 j}=R_{2 j}=1\right)\right. \\
& \left.-2 \operatorname{Cov}\left(Y_{t j}, Y_{c j} \mid R_{1 j}=R_{2 j}=1\right)\right\} \\
= & \frac{1}{N_{t c}-1} \operatorname{Var}\left(Y_{t j}-Y_{c j} \mid R_{1 j}=R_{2 j}=1\right) \\
\widehat{\mathbb{V}}^{\text {Adj-Neyman }}\left(\hat{\tau}_{U}\right) \equiv & \frac{1}{N_{t}-1} \operatorname{Var}\left(Y_{i j} \mid X_{i j}=1, R_{i j}=1\right)+\frac{1}{N_{c}-1} \operatorname{Var}\left(Y_{i j} \mid X_{i j}=0, R_{i j}=1\right) \\
& -\frac{2 N_{t c}^{2}}{N_{t} N_{c}\left(N_{t c}-1\right)} \operatorname{Cov}\left(Y_{t j}, Y_{c j} \mid R_{1 j}=R_{2 j}=1\right) .
\end{aligned}
$$

It turns out that the adjusted Neyman variance estimator of $\hat{\tau}_{F}$ is reduced to what scholars recommend (Gerber and Green 2012, 77; Imai 2008, 4861; Imbens and Rubin 2015, 227). This paper extends it to $\hat{\tau}_{P}$ and, in particular, $\hat{\tau}_{U}$. I now derive the properties of these variance estimators.

PROPOSITION 3 (Bias of the Neyman Variance Estimators: FS)

(1) Under Assumption 1,

$$
\mathbb{E}\left\{\widehat{\mathbb{V}}^{\text {Neyman }}\left(\hat{\tau}_{F}\right)\right\}-\mathbb{V}^{2}\left(\hat{\tau}_{F}\right)=\frac{1}{N-1}\left[\operatorname{Var}\left\{\beta_{i j}(1)\right\}+\operatorname{Var}\left\{\beta_{i j}(0)\right\}\right]-\frac{2}{N} \operatorname{Cov}\left\{\omega_{i j}(1), \omega_{i j}(0)\right\}
$$

(2) Under Assumption 3 and $N_{t c} \geq 2$,

$$
\begin{aligned}
\mathbb{E}\left\{\widehat{\mathbb{V}}^{\text {Neyman }}\left(\hat{\tau}_{P}\right)\right\}-\mathbb{V}^{2}\left(\hat{\tau}_{P}\right)= & \frac{1}{N_{t c}-1}\left[\operatorname{Var}\left\{\beta_{i j}(1) \mid R_{1 j}(1)=R_{2 j}(1)=R_{1 j}(0)=R_{2 j}(0)=1\right\}\right. \\
& \left.+\operatorname{Var}\left\{\beta_{i j}(0) \mid R_{1 j}(1)=R_{2 j}(1)=R_{1 j}(0)=R_{2 j}(0)=1\right\}\right] \\
& -\frac{2}{N_{t c}} \operatorname{Cov}\left\{\omega_{i j}(1), \omega_{i j}(0) \mid R_{1 j}(1)=R_{2 j}(1)=R_{1 j}(0)=R_{2 j}(0)=1\right\} .
\end{aligned}
$$

(3) Under Assumption 2 and $N_{t}, N_{c} \geq 2$,

$$
\begin{aligned}
\mathbb{E}\left\{\widehat{\mathbb{V}}^{\text {Neyman }}\left(\hat{\tau}_{U}\right)\right\}-\mathbb{V}^{2}\left(\hat{\tau}_{U}\right)= & \frac{1}{N_{t}-1} \operatorname{Var}\left\{\beta_{i j}(1) \mid R_{i j}(1)=1\right\}+\frac{1}{N_{c}-1} \operatorname{Var}\left\{\beta_{i j}(0) \mid R_{i j}(0)=1\right\} \\
& -\frac{2 N_{t c}}{N_{t} N_{c}} \operatorname{Cov}\left\{\omega_{i j}(1), \omega_{i j}(0) \mid R_{1 j}(1)=R_{2 j}(1)=R_{1 j}(0)=R_{2 j}(0)=1\right\} .
\end{aligned}
$$

PROPOSITION 4 (Bias of the Adjusted Neyman Variance Estimators: FS)

(1) Under Assumption 1,

$$
\mathbb{E}\left\{\widehat{\mathbb{V}}^{\text {Adj-Neyman }}\left(\hat{\tau}_{F}\right)\right\}-\mathbb{V}^{2}\left(\hat{\tau}_{F}\right)=\frac{1}{N-1} \operatorname{Var}\left\{\beta_{i j}(1)-\beta_{i j}(0)\right\} \geq 0
$$


(2) Under Assumption 3 and $N_{t c} \geq 2$,

$$
\begin{aligned}
& \mathbb{E}\left\{\widehat{\mathbb{V}}^{\text {Adj-Neyman }}\left(\hat{\tau}_{P}\right)\right\}-\mathbb{V}^{2}\left(\hat{\tau}_{P}\right) \\
= & \frac{1}{N_{t c}-1} \operatorname{Var}\left\{\beta_{i j}(1)-\beta_{i j}(0) \mid R_{1 j}(1)=R_{2 j}(1)=R_{1 j}(0)=R_{2 j}(0)=1\right\} \geq 0 .
\end{aligned}
$$

(3) Under Assumption 2 and $N_{t c} \geq 2$,

$$
\begin{aligned}
& \mathbb{E}\left\{\widehat{\mathbb{V}}^{\text {Adj-Neyman }}\left(\hat{\tau}_{U}\right)\right\}-\mathbb{V}^{2}\left(\hat{\tau}_{U}\right) \\
= & \frac{1}{N_{t}-1} \operatorname{Var}\left\{\beta_{i j}(1) \mid R_{i j}(1)=1\right\}+\frac{1}{N_{c}-1} \operatorname{Var}\left\{\beta_{i j}(0) \mid R_{i j}(0)=1\right\} \\
& -\frac{2 N_{t c}^{2}}{N_{t} N_{c}\left(N_{t c}-1\right)} \operatorname{Cov}\left\{\beta_{i j}(1), \beta_{i j}(0) \mid R_{1 j}(1)=R_{2 j}(1)=R_{1 j}(0)=R_{2 j}(0)=1\right\} .
\end{aligned}
$$

A merit of $\widehat{\mathbb{V}}^{\text {Adj-Neyman }}\left(\hat{\tau}_{F}\right)$ and $\widehat{\mathbb{V}}^{\text {Adj-Neyman }}\left(\hat{\tau}_{P}\right)$ is that they cannot have negative bias (and thus they are conservative). By contrast, the other variance estimators can be downwardly biased.

\section{Super-Population}

\subsection{Setting}

Following Imai (2008) and Imbens and Rubin (2015, chs. 6 and 10, esp. pp. 109 and 229), this paper supposes that the above $N$ pairs of a finite sample are drawn from a super-population that is composed of $N^{*}(>N)$ pairs, with $N^{*}$ large, but countable. I define super-population variables and operators in the same way as the corresponding finite sample variables and operators, and denote them by adding superscript * to each term. In particular, the main estimand of this section, the super-population ATE, is denoted by $\tau^{*} \equiv E^{*}\left\{Y_{i^{*} j^{*}}^{*}(1)-Y_{i^{*} j^{*}}^{*}(0)\right\} \equiv \frac{1}{2 N^{*}} \sum_{j^{*}=1}^{N^{*}} \sum_{i^{*}=1}^{2}\left\{Y_{i^{*} j^{*}}^{*}(1)-\right.$ $\left.Y_{i^{*} j^{*}}^{*}(0)\right\}$.

I assume random sampling of pairs. In addition, the following three assumptions of potential responses are optional in the same spirit as the finite sample case. SP stands for the "superpopulation."

ASSUMPTION $1^{*}$ (No Attrition: SP) $\forall i^{*}, j^{*}, R_{i^{*} j^{*}}^{*}(1)=R_{i^{*} j^{*}}^{*}(0)=1$.

AsSUMPtION 2* (Unitwise Matched Attrition: SP) $\forall j^{*}, R_{1 j^{*}}^{*}(1)=R_{2 j^{*}}^{*}(1), R_{1 j^{*}}^{*}(0)=R_{2 j^{*}}^{*}(0)$.

AssumPtION $3^{*}$ (Pairwise Matched Attrition: SP) $\forall j^{*}, R_{1 j^{*}}^{*}(1) R_{2 j^{*}}^{*}(0)=R_{1 j^{*}}^{*}(0) R_{2 j^{*}}^{*}(1)$.

Note that unlike the case of a finite sample, in the super-population perspective, $N_{t}, N_{c}$, and $N_{t c}$ are not constant across sampling of pairs even under Assumption $2^{*}$ or $3^{*}$.

\subsection{Bias}

In this section, the operator $\mathbb{E}^{*}(\cdot)$ takes expectation not only over the random assignment of the treatment but also over the random sampling of the pairs. Define

$$
\bar{\beta}^{*}\left(x \mid r_{1}, r_{0}\right) \equiv E^{*}\left\{\beta_{i^{*} j^{*}}^{*}(x) \mid R_{1 j^{*}}^{*}(1)=R_{2 j^{*}}^{*}(1)=r_{1}, R_{1 j^{*}}^{*}(0)=R_{2 j^{*}}^{*}(0)=r_{0}\right\}
$$

The bias of each ATE estimator is as follows.

PROPOSITION $1^{*}$ (Bias of ATE Estimators: SP)

(1) Under Assumption $7^{*}, \mathbb{E}^{*}\left(\hat{\tau}_{F}\right)-\tau^{*}=0$. 
(2) Under Assumption 3*,

$$
\mathbb{E}^{*}\left(\hat{\tau}_{P} \mid N_{t c} \geq 1\right)-\tau^{*}=E^{*}\left\{\beta_{i^{*} j^{*}}^{*}(1)-\beta_{i^{*} j^{*}}^{*}(0) \mid R_{1 j^{*}}^{*}(1)=R_{2 j^{*}}^{*}(1)=R_{1 j^{*}}^{*}(0)=R_{2 j^{*}}^{*}(0)=1\right\}
$$

(3) Under Assumption 2*,

$$
\begin{aligned}
& \mathbb{E}^{*}\left(\hat{\tau}_{U} \mid N_{t}, N_{c} \geq 1\right)-\tau^{*} \\
= & \left\{\mathbb{E}^{*}\left(\frac{N_{t c}}{N_{t}} \mid N_{t}, N_{c} \geq 1\right) \bar{\beta}^{*}(1 \mid 1,1)+\mathbb{E}^{*}\left(\frac{N_{t}-N_{t c}}{N_{t}} \mid N_{t}, N_{c} \geq 1\right) \bar{\beta}^{*}(1 \mid 1,0)\right\} \\
& -\left\{\mathbb{E}^{*}\left(\frac{N_{t c}}{N_{c}} \mid N_{t}, N_{c} \geq 1\right) \bar{\beta}^{*}(0 \mid 1,1)+\mathbb{E}^{*}\left(\frac{N_{c}-N_{t c}}{N_{c}} \mid N_{t}, N_{c} \geq 1\right) \bar{\beta}^{*}(0 \mid 0,1)\right\} .
\end{aligned}
$$

As in the case of a finite sample, unless we assume ignorable attrition, not only $\hat{\tau}_{U}$ but also $\hat{\tau}_{P}$ is biased for $\tau^{*}$. It also holds that $\mathbb{E}^{*}\left(\hat{\tau}_{P}\right)-\tau_{P}^{*}=0$. Furthermore, note that in general,

$$
\mathbb{E}^{*}\left(\hat{\tau}_{U} \mid N_{t}, N_{c} \geq 1\right)-\tau^{*} \neq E^{*}\left\{\beta_{i^{*} j^{*}}^{*}(1) \mid R_{i^{*} j^{*}}^{*}(1)=1\right\}-E^{*}\left\{\beta_{i^{*} j^{*}}^{*}(0) \mid R_{i^{*} j^{*}}^{*}(0)=1\right\}
$$

because

$$
\mathbb{E}^{*}\left(\frac{N_{t c}}{N_{t}} \mid N_{t}, N_{c} \geq 1\right) \geq \frac{N_{t c}^{*}}{N_{t}^{*}}, \quad \mathbb{E}^{*}\left(\frac{N_{t c}}{N_{c}} \mid N_{t}, N_{c} \geq 1\right) \geq \frac{N_{t c}^{*}}{N_{c}^{*}}
$$

\subsection{Variance}

In this section, $\operatorname{Var}^{*}(\cdot)$ and $\operatorname{Cov}^{*}(\cdot, \cdot)$ denote the super-population variance and covariance, respectively, and the operators $\mathbb{V}^{*}(\cdot)$ and $\mathbb{V}^{*}(\cdot, \cdot)$ take variance and covariance, respectively, not only over the random assignment of the treatment but also over the random sampling of the pairs. In addition, I assume that under Assumption $3^{*}$, either $\lim _{N^{*} \rightarrow \infty} N_{t c}^{*}=\infty$ or $\lim _{N^{*} \rightarrow \infty} N_{t c}^{*}<2$ holds. I also assume that under Assumption 2 ${ }^{*}$, the same conditions hold not only for $N_{t c}^{*}$ but also for $N_{t}^{*}-N_{t c}^{*}$ and $N_{c}^{*}-N_{t c}^{*}$.

Below, I derive the super-population variance of the three ATE estimators in the limit. Note that I increase $N^{*}$, not $N$.

\section{PROPOSITION 2* (Variance of ATE Estimators: SP)}

(1) Under Assumption 1*,

$$
\lim _{N^{*} \rightarrow \infty} \mathbb{V}^{2 *}\left(\hat{\tau}_{F}\right)=\frac{1}{N}\left[\operatorname{Var}^{*}\left\{\beta_{i^{*} j^{*}}^{*}(1)-\beta_{i^{*} j^{*}}^{*}(0)\right\}+\operatorname{Var}^{*}\left\{\omega_{i^{*} j^{*}}^{*}(1)+\omega_{i^{*} j^{*}}^{*}(0)\right\}\right] .
$$

(2) Under Assumption 3*,

$$
\begin{aligned}
& \lim _{N^{*} \rightarrow \infty} \mathbb{V}^{2 *}\left(\hat{\tau}_{P} \mid N_{t c} \geq 1\right) \\
= & \mathbb{E}^{*}\left(\frac{1}{N_{t c}} \mid N_{t c} \geq 1\right)\left[\operatorname{Var}^{*}\left\{\beta_{i^{*} j^{*}}^{*}(1)-\beta_{i^{*} j^{*}}^{*}(0) \mid R_{1 j^{*}}^{*}(1)=R_{2 j^{*}}^{*}(1)=R_{1 j^{*}}^{*}(0)=R_{2 j^{*}}^{*}(0)=1\right\}\right. \\
& \left.+\operatorname{Var}^{*}\left\{\omega_{i^{*} j^{*}}^{*}(1)+\omega_{i^{*} j^{*}}^{*}(0) \mid R_{1 j^{*}}^{*}(1)=R_{2 j^{*}}^{*}(1)=R_{1 j^{*}}^{*}(0)=R_{2 j^{*}}^{*}(0)=1\right\}\right] .
\end{aligned}
$$

(3) Under Assumption 2*,

$$
\begin{aligned}
& \lim _{N^{*} \rightarrow \infty} \mathbb{V}^{2 *}\left(\hat{\tau}_{U} \mid N_{t}, N_{c} \geq 1\right) \\
& =\left(\left[\mathbb{E}^{*}\left(\frac{N_{t c}}{N_{t}^{2}} \mid N_{t}, N_{c} \geq 1\right)\left\{\tilde{\beta}^{2 *}(1 \mid 1,1)+\tilde{\omega}^{2 *}(1 \mid 1,1)\right\}\right.\right.
\end{aligned}
$$




$$
\begin{aligned}
& \left.+\mathbb{E}^{*}\left(\frac{N_{t}-N_{t c}}{N_{t}^{2}} \mid N_{t}, N_{c} \geq 1\right)\left\{\tilde{\beta}^{2 *}(1 \mid 1,0)+\tilde{\omega}^{2 *}(1 \mid 1,0)\right\}\right] \\
& +\left[\mathbb{E}^{*}\left(\frac{N_{t c}}{N_{c}^{2}} \mid N_{t}, N_{c} \geq 1\right)\left\{\tilde{\beta}^{2 *}(0 \mid 1,1)+\tilde{\omega}^{2 *}(0 \mid 1,1)\right\}\right. \\
& \left.+\mathbb{E}^{*}\left(\frac{N_{c}-N_{t c}}{N_{c}^{2}} \mid N_{t}, N_{c} \geq 1\right)\left\{\tilde{\beta}^{2 *}(0 \mid 0,1)+\tilde{\omega}^{2 *}(0 \mid 0,1)\right\}\right] \\
& -2 \mathbb{E}^{*}\left(\frac{N_{t c}}{N_{t} N_{c}} \mid N_{t}, N_{c} \geq 1\right)\left[\operatorname{Cov}^{*}\left\{\beta_{i^{*} j^{*}}^{*}(1), \beta_{i^{*} j^{*}}^{*}(0) \mid R_{1 j^{*}}^{*}(1)=R_{2 j^{*}}^{*}(1)=R_{1 j^{*}}^{*}(0)=R_{2 j^{*}}^{*}(0)=1\right\}\right. \\
& \left.\left.-\operatorname{Cov}^{*}\left\{\omega_{i^{*} j^{*}}^{*}(1), \omega_{i^{*} j^{*}}^{*}(0) \mid R_{1 j^{*}}^{*}(1)=R_{2 j^{*}}^{*}(1)=R_{1 j^{*}}^{*}(0)=R_{2 j^{*}}^{*}(0)=1\right\}\right]\right) \\
& +\left[\mathbb{V}^{2 *}\left(\frac{N_{t c}}{N_{t}} \mid N_{t}, N_{c} \geq 1\right)\left\{\bar{\beta}^{*}(1 \mid 1,1)-\bar{\beta}^{*}(1 \mid 1,0)\right\}^{2}\right. \\
& +\mathbb{V}^{2 *}\left(\frac{N_{t c}}{N_{c}} \mid N_{t}, N_{c} \geq 1\right)\left\{\bar{\beta}^{*}(0 \mid 1,1)-\bar{\beta}^{*}(0 \mid 0,1)\right\}^{2} \\
& \left.-2 \mathbb{V}^{*}\left(\frac{N_{t c}}{N_{t}}, \frac{N_{t c}}{N_{c}} \mid N_{t}, N_{c} \geq 1\right)\left\{\bar{\beta}^{*}(1 \mid 1,1)-\bar{\beta}^{*}(1 \mid 1,0)\right\}\left\{\bar{\beta}^{*}(0 \mid 1,1)-\bar{\beta}^{*}(0 \mid 0,1)\right\}\right],
\end{aligned}
$$

where

$$
\begin{aligned}
& \tilde{\beta}^{2 *}\left(x \mid r_{1}, r_{0}\right)=\operatorname{Var}^{*}\left\{\beta_{i^{*} j^{*}}^{*}(x) \mid R_{1 j^{*}}^{*}(1)=R_{2 j^{*}}^{*}(1)=r_{1}, R_{1 j^{*}}^{*}(0)=R_{2 j^{*}}^{*}(0)=r_{0}\right\} \\
& \tilde{\omega}^{2 *}\left(x \mid r_{1}, r_{0}\right)=\operatorname{Var}^{*}\left\{\omega_{i^{*} j^{*}}^{*}(x) \mid R_{1 j^{*}}^{*}(1)=R_{2 j^{*}}^{*}(1)=r_{1}, R_{1 j^{*}}^{*}(0)=R_{2 j^{*}}^{*}(0)=r_{0}\right\} .
\end{aligned}
$$

Like the case of a finite sample, $\mathbb{V}^{2 *}\left(\hat{\tau}_{U} \mid N_{t c} \geq 1\right)$ can be larger than $\mathbb{V}^{2 *}\left(\hat{\tau}_{P} \mid N_{t c} \geq 1\right)$.

\subsection{Variance Estimator}

Finally, I show the super-population biases of the two variance estimators in the limit.

PROPOSITION 3* (Bias of the Neyman Variance Estimators: SP)

(1) Under Assumption 1*,

$$
\lim _{N^{*} \rightarrow \infty}\left[\mathbb{E}^{*}\left\{\widehat{\mathbb{V}}^{\text {Neyman }}\left(\hat{\tau}_{F}\right)\right\}-\mathbb{V}^{2 *}\left(\hat{\tau}_{F}\right)\right]=\frac{2}{N}\left[\operatorname{Cov}^{*}\left\{\beta_{i^{*} j^{*}}^{*}(1), \beta_{i^{*} j^{*}}^{*}(0)\right\}-\operatorname{Cov}^{*}\left\{\omega_{i^{*} j^{*}}^{*}(1), \omega_{i^{*} j^{*}}^{*}(0)\right\}\right]
$$

(2) Under Assumption 3*,

$$
\begin{aligned}
& \lim _{N^{*} \rightarrow \infty}\left[\mathbb{E}^{*}\left\{\widehat{\mathbb{V}}^{\text {Neyman }}\left(\hat{\tau}_{P}\right) \mid N_{t c} \geq 2\right\}-\mathbb{V}^{2 *}\left(\hat{\tau}_{P} \mid N_{t c} \geq 2\right)\right] \\
= & 2 \mathbb{E}^{*}\left(\frac{1}{N_{t c}} \mid N_{t c} \geq 2\right)\left[\operatorname{Cov}^{*}\left\{\beta_{i^{*} j^{*}}^{*}(1), \beta_{i^{*} j^{*}}^{*}(0) \mid R_{1 j^{*}}^{*}(1)=R_{2 j^{*}}^{*}(1)=R_{1 j^{*}}^{*}(0)=R_{2 j^{*}}^{*}(0)=1\right\}\right. \\
& \left.-\operatorname{Cov}^{*}\left\{\omega_{i^{*} j^{*}}^{*}(1), \omega_{i^{*} j^{*}}^{*}(0) \mid R_{1 j^{*}}^{*}(1)=R_{2 j^{*}}^{*}(1)=R_{1 j^{*}}^{*}(0)=R_{2 j^{*}}^{*}(0)=1\right\}\right] .
\end{aligned}
$$

(3) Under Assumption 2*,

$$
\begin{aligned}
\lim _{N^{*} \rightarrow \infty}\left[\mathbb{E}^{*}\left\{\widehat{\mathbb{V}}^{\text {Neyman }}\left(\hat{\tau}_{U}\right) \mid N_{t}, N_{c} \geq 2\right\}-\mathbb{V}^{2 *}\left(\hat{\tau}_{U} \mid N_{t}, N_{c} \geq 2\right)\right] \\
=2 \mathbb{E}^{*}\left(\frac{N_{t c}}{N_{t} N_{c}} \mid N_{t}, N_{c} \geq 2\right)\left[\operatorname{Cov}^{*}\left\{\beta_{i^{*} j^{*}}^{*}(1), \beta_{i^{*} j^{*}}^{*}(0) \mid R_{1 j^{*}}^{*}(1)=R_{2 j^{*}}^{*}(1)=R_{1 j^{*}}^{*}(0)=R_{2 j^{*}}^{*}(0)=1\right\}\right. \\
\left.-\operatorname{Cov}^{*}\left\{\omega_{i^{*} *^{*}}^{*}(1), \omega_{i^{*} j^{*}}^{*}(0) \mid R_{1 j^{*}}^{*}(1)=R_{2 j^{*}}^{*}(1)=R_{1 j^{*}}^{*}(0)=R_{2 j^{*}}^{*}(0)=1\right\}\right] \\
+\left[\left[\mathbb{E}^{*}\left\{\frac{N_{t c}\left(N_{t}-N_{t c}\right)}{N_{t}^{2}\left(N_{t}-1\right)} \mid N_{t}, N_{c} \geq 2\right\}-\mathbb{V}^{2 *}\left(\frac{N_{t c}}{N_{t}} \mid N_{t}, N_{c} \geq 2\right)\right]\left\{\bar{\beta}^{*}(1 \mid 1,1)-\bar{\beta}^{*}(1 \mid 1,0)\right\}^{2}\right.
\end{aligned}
$$




$$
\begin{aligned}
& +\left[\mathbb{E}^{*}\left\{\frac{N_{t c}\left(N_{c}-N_{t c}\right)}{N_{c}^{2}\left(N_{c}-1\right)} \mid N_{t}, N_{c} \geq 2\right\}-\mathbb{V}^{2 *}\left(\frac{N_{t c}}{N_{c}} \mid N_{t}, N_{c} \geq 2\right)\right]\left\{\bar{\beta}^{*}(0 \mid 1,1)-\bar{\beta}^{*}(0 \mid 0,1)\right\}^{2} \\
& +2 \mathbb{V}^{*}\left(\frac{N_{t c}}{N_{t}}, \frac{N_{t c}}{N_{c}} \mid N_{t}, N_{c} \geq 2\right)\left\{\bar{\beta}^{*}(1 \mid 1,1)-\bar{\beta}^{*}(1 \mid 1,0)\right\}\left\{\bar{\beta}^{*}(0 \mid 1,1)-\bar{\beta}^{*}(0 \mid 0,1)\right\}
\end{aligned}
$$

PROPOSITION 4* (Bias of the Adjusted Neyman Variance Estimators: SP)

(1) Under Assumption 1*,

$$
\lim _{N^{*} \rightarrow \infty}\left[\mathbb{E}^{*}\left\{\widehat{\mathbb{V}}^{\text {Adj-Neyman }}\left(\hat{\tau}_{F}\right)\right\}-\mathbb{V}^{2 *}\left(\hat{\tau}_{F}\right)\right]=0
$$

(2) Under Assumption 3*,

$$
\lim _{N^{*} \rightarrow \infty}\left[\mathbb{E}^{*}\left\{\widehat{\mathbb{V}}^{\text {Adj-Neyman }}\left(\hat{\tau}_{P}\right) \mid N_{t c} \geq 2\right\}-\mathbb{V}^{2 *}\left(\hat{\tau}_{P} \mid N_{t c} \geq 2\right)\right]=0 .
$$

(3) Under Assumption 2*,

$$
\begin{aligned}
\lim _{N^{*} \rightarrow \infty}\left[\mathbb{E}^{*}\left\{\widehat{\mathbb{V}}^{\text {Adj-Neyman }}\left(\hat{\tau}_{U}\right) \mid N_{t c} \geq 2\right\}-\mathbb{V}^{2 *}\left(\hat{\tau}_{U} \mid N_{t c} \geq 2\right)\right] \\
=\left[\mathbb{E}^{*}\left\{\frac{N_{t c}\left(N_{t}-N_{t c}\right)}{N_{t}^{2}\left(N_{t}-1\right)} \mid N_{t c} \geq 2\right\}-\mathbb{V}^{2 *}\left(\frac{N_{t c}}{N_{t}} \mid N_{t c} \geq 2\right)\right]\left\{\bar{\beta}^{*}(1 \mid 1,1)-\bar{\beta}^{*}(1 \mid 1,0)\right\}^{2} \\
+\left[\mathbb{E}^{*}\left\{\frac{N_{t c}\left(N_{c}-N_{t c}\right)}{N_{c}^{2}\left(N_{c}-1\right)} \mid N_{t c} \geq 2\right\}-\mathbb{V}^{2 *}\left(\frac{N_{t c}}{N_{c}} \mid N_{t c} \geq 2\right)\right]\left\{\bar{\beta}^{*}(0 \mid 1,1)-\bar{\beta}^{*}(0 \mid 0,1)\right\}^{2} \\
+2 \mathbb{V}^{*}\left(\frac{N_{t c}}{N_{t}}, \frac{N_{t c}}{N_{c}} \mid N_{t c} \geq 2\right)\left\{\bar{\beta}^{*}(1 \mid 1,1)-\bar{\beta}^{*}(1 \mid 1,0)\right\}\left\{\bar{\beta}^{*}(0 \mid 1,1)-\bar{\beta}^{*}(0 \mid 0,1)\right\} .
\end{aligned}
$$

Proposition $4^{*}(2)$ is new to my knowledge and surprising. Even if we do not assume ignorable attrition, the adjusted Neyman variance estimator is unbiased for the super-population variance of the PDE. Here is an intuitive explanation: If we regard always-reporting pairs as an alternative super-population and apply Proposition $4^{*}(1)$ to it, we obtain Proposition $4^{*}(2)$. In a nutshell, $\widehat{\mathbb{V}}^{\text {Adj-Neyman }}\left(\hat{\tau}_{P}\right)$ corresponds to the design of pairwise randomization. On the other hand, the bias directions of the other variance estimators are unknown.

\section{Conclusion}

Nonignorable attrition in pairwise randomized experiments has attracted less attention than it should. This paper shows that the UDE and the PDE are biased (Propositions 1 and $1^{*}$ ). Nonetheless, a practical advice of this paper is simple: use the PDE rather than the UDE. The reasons are summarized as follows:

1. The PDE can be regarded as a kind of local average treatment effect for always-reporting pairs under a milder assumption than the UDE.

2. As compared with the UDE, the PDE is not necessarily less efficient. (Propositions 2 and $2^{*}$ )

3. The adjusted Neyman variance estimator for the PDE is conservative in a finite sample and unbiased in a super-population, which is not the case for the UDE. (Propositions 4 and $4^{*}$ )

Finally, the Neyman variance estimator can have either positive or negative bias for both ATE estimators (Propositions 3 and $3^{*}$ ) and thus is not recommended.

\section{Acknowledgments}

Earlier versions, whose titles were "Blocking Reduces, if not Removes, Attrition Bias" or "Missing Data under the Matched-Pair Design: A Practical Guide," were presented at the Workshop on Directional Statistics, the Institute of Statistical Mathematics (ISM), Tokyo, July 5, 2012, the Asian Political Methodology Meeting (APMM), Tokyo Institute of Technology, January 6-7, 2014, the Annual 
Meeting of the Midwest Political Science Association (MPSA), Chicago, April 16-19, 2015, the Annual Summer Meeting of Society for Political Methodology, Rochester, July 23-25, 2015, International Methods Colloquium, September 26, 2015, the Annual Meeting of the Japanese Political Science Association, Chiba University, October 10, 2015, the APMM, University of Sydney, January 9-11, 2017, the Annual Meeting of the MPSA, Chicago, April 6-9, 2017, the Japanese Economic Association Spring Meeting, Ritsumeikan University, June 24-25, 2017, and workshops at Dartmouth College, ISM, Osaka University, University of California, San Diego, and Washington University in St. Louis. I appreciate comments from Raymond Duch, Justin Esarey, Mototsugu Fukushige, Jeff Gill, Donald Green, Yoichi Hizen, Yusaku Horiuchi, Kosuke Imai, Luke Keele, Gary King, Dean Lacy, Charles McClean, Ryan T. Moore, Brendan Nyhan, Fredrik Sävje, Keith Schnakenberg, Susumu Shikano, Patrick Tucker, and Takahide Yanagi.

\section{Funding}

This work was supported by Japan Society for the Promotion of Science [grant numbers KAKENHI JP26285031, JP16K13340]; and Computer Centre of Gakushuin University [no grant number].

\section{Supplementary Material}

For supplementary material accompanying this paper, please visit https://doi.org/10.1017/pan. 2020.51.

\section{Data Availability Statement}

The replication materials for the supplementary material of this paper can be found at Fukumoto (2020).

\section{Conflict of Interest}

There is no conflict of interest to disclose.

\section{References}

Allison, P. D. 2002. Missing Data: Quantitative Applications in the Social Sciences. Thousand Oaks: SAGE Publications.

Donner, A., and N. Klar. 2000. Design and Analysis of Cluster Randomization Trials in Health Research. London: Arnold.

Dunning, T. 2011. “Does Blocking Reduce Attrition Bias?” Experimental Political Scientist: Newsletter of the APSA Experimental Section 2(1):12-16.

Frangakis, C. E., and D. B. Rubin. 2002. "Principal Stratification in Causal Inference." Biometrics 58(1): 21-29.

Fukumoto, K.. 2020. "Replication Data for: Nonignorable Attrition in Pairwise Randomized Experiments." https://doi.org/10.7910/DVN/O9WE06, Harvard Dataverse, V1.

Gerber, A. S., and D. P. Green. 2012. Field Experiments: Design, Analysis, and Interpretation. New York: W. W. Norton \& Company.

Glennerster, R., and K. Takavarasha. 2013. Running Randomized Evaluations. Princeton: Princeton University Press.

Hayes, R. J., and L. H. Moulton. 2009. Cluster Randomised Trials. Boca Raton: Chapman \& Hall/CRC.

Imai, K. 2008. "Variance Identification and Efficiency Analysis in Randomized Experiments under the Matched-Pair Design". Statistics in Medicine 27(24): 4857-4873.

Imai, K., and Z. Jiang. 2018. "A Sensitivity Analysis for Missing Outcomes due to Truncation by Death under the Matched-Pairs Design." Statistics in Medicine 37(20): 2907-2922.

Imai, K., G. King, and C. Nall 2009. "The Essential Role of Pair Matching in Cluster-Randomized Experiments, with Application to the Mexican Universal Health Insurance Evaluation." Statistical Science 24(1): 29-53.

Imbens, G. W., and D. B. Rubin. 2015. Causal Inference for Statistics, Social, and Biomedical Sciences. New York: Cambridge University Press.

Little, R. J. A., and D. B. Rubin. 2002. Statistical Analysis with Missing Data. 2nd Edn. Hoboken: John Wiley \& Sons.

Lynn, H. S., and C. E. McCulloch. 1992. "When Does It Pay to Break the Matches for Analysis of a Matched-Pairs Design?” Biometrics 48(2): 397-409. 